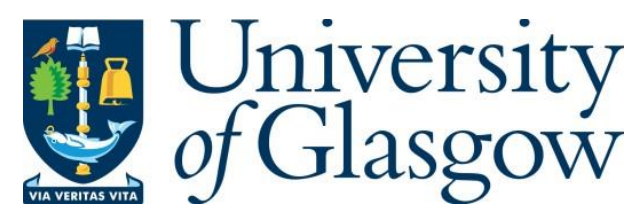

Lloret-Cabot, M., Pineda, J. A. and Sheng, D. (2018) Numerical Implementation of a Critical State Model for Soft Rocks. In: 2nd Pan-American Conference on Unsaturated Soils (PANAM-UNSAT 2017), Dallas, TX, USA, 12-15 Nov 2017, pp. 236-246. ISBN 9780784481684.

There may be differences between this version and the published version. You are advised to consult the publisher's version if you wish to cite from it.

http://eprints.gla.ac.uk/151078/

Deposited on: 6 November 2017

Enlighten - Research publications by members of the University of Glasgow http://eprints.gla.ac.uk 


\title{
Numerical Implementation of a Critical State Model for Soft Rocks
}

\author{
Martí Lloret-Cabot, Ph.D., ${ }^{1,2}$ Jubert A. Pineda, PhD., \\ and Daichao Sheng ${ }^{3}$
}

\author{
${ }^{1}$ University of Glasgow, Division of Infrastructure and Environment, Rankine Building, \\ Glasgow, G12 8LT; e-mail: marti.lloret-cabot@ glasgow.ac.uk \\ ${ }^{2}$ University of Newcastle, Faculty of Engineering and Built Environment, EA Building, \\ Callaghan, 2308 NSW; e-mail: jubert.pineda@newcastle.edu.au \\ ${ }^{3}$ University of Newcastle, Faculty of Engineering and Built Environment, EA Building, \\ Callaghan, 2308 NSW; e-mail: daichao.sheng@newcastle.edu.au
}

\begin{abstract}
This paper details the basic tasks for the numerical implementation of a simple elasto-plastic critical state model for bonded materials (i.e. soft rocks-hard soils) into the finite element program SNAC developed at the University of Newcastle in Australia. The first task described focusses on the derivation of the incremental constitutive relationships used to represent the mechanical response of a bonded/cemented material under saturated conditions. The second task presents how these stress-strain relations can be numerically integrated using an explicit substepping scheme with automatic error control. The third task concentrates on the verification of the substepping algorithm proposed. The model used to represent the saturated mechanical response of a bonded material combines the modified Cam clay with the constitutive relationships for cemented materials proposed in Gens \& Nova (1993), but incorporates some flexibility on the degradation law adopted. The role of suction and other relevant aspects of unsaturated behaviour are also discussed at the end of the paper.
\end{abstract}

\section{INTRODUCTION}

This paper presents a simple constitutive model to represent the mechanical response of saturated soft rocks-hard soils. In essence, the representation of the mechanical behaviour of these bonded or cemented materials is achieved in the paper by combining the modified Cam clay, MCC, (Roscoe \& Burland 1968) with the constitutive relationships for bonded materials proposed in Gens \& Nova (1993). A small variation on how cementation is destroyed under mechanical and environmental actions is adopted in the paper to test the validity of the degradation law proposed by Pineda et al. (2014a). To understand the performance of this simple model, its corresponding incremental stress-strain relationships are integrated using an explicit substepping formulation 
with automatic error control (Sloan 1987; Sloan et al. 2001). The paper demonstrates how useful is to use this small program to check the numerical response for simple stress paths whose behaviour is known in advance. At this stage of the implementation process, it is also convenient to verify other theoretical and computational aspects of the integration scheme used. Once the correctness of the integration scheme has been checked, it is simple to include this small driver into a larger finite element program. For this purpose, this research uses the finite element program SNAC developed at the University of Newcastle in Australia (Abbo 1997; Sloan and Abbo 1999; Sheng et al. 2003).

\section{MODELLING BONDED MATERIALS: SATURATED CONDITIONS}

Soft rocks-hard soils are geotechnical materials sharing mechanical features from rocks and soils and are, therefore, often referred to as transitional materials. To represent their mechanical behaviour, the existence of internal cementations/bonds around inter-particle (or inter-aggregate) contacts can be idealized as part of their internal structure acting as a cementation. The occurrence of this cementation in the material has an influence on its mechanical response that is gradually lost with the reduction (or degradation) of cementation occurring during application of mechanical and environmental actions. In this paper, the constitutive laws proposed in Gens and Nova (1993) are incorporated into the MCC model to represent the saturated mechanical response of these transitional materials. A small modification on the way in which the effect of bonding is reduced during mechanical yielding is introduced in the paper to test the numerical performance of a novel degradation law proposed by Pineda et al. (2014a). This new degradation law was obtained from long-term relative humidity cycling tests carried out on Lilla claystone (Spain). It is therefore important to test first its numerical performance for saturated conditions, in view of any future extension of the saturated model to include unsaturated stress states. In this context, it is also important to ensure at this stage, that the saturated framework adopted is also a suitable platform from where the representation of the transitions between saturated and unsaturated states can be modelled (Lloret-Cabot et al. 2017a) and that such representation is done consistently with the elasto-plastic critical state theory typically adopted to model saturated conditions (Lloret-Cabot et al. 2017b).

Stress state variables. For stress states that correspond to the triaxial test, the stress state variables of the model are the saturated mean effective stress $p^{\prime}$ and the deviator stress $q$ :

$$
\begin{aligned}
& p^{\prime}=p-u_{\mathrm{w}} \\
& q=\sigma_{1}-\sigma_{2}
\end{aligned}
$$

where $p$ is mean total stress, $u_{\mathrm{w}}$ is pore water pressure, $\sigma_{1}$ and $\sigma_{2}$ are, respectively, major and minor principal total stresses.

Elastic behaviour. Elastic components of $d \varepsilon_{\mathrm{v}}$ and $\mathrm{d} \varepsilon_{\mathrm{q}}$ are assumed to be given by: 
$d \varepsilon_{\mathrm{v}}^{\mathrm{e}}=\frac{\kappa d p^{\prime}}{v p^{\prime}}$

$d \varepsilon_{\mathrm{q}}^{\mathrm{e}}=\frac{d q}{3 G}$

where $v$ is the specific volume, $\kappa$ is the gradient of elastic unloading-reloading lines in the $v: \ln p^{\prime}$ plane and $G$ is the elastic shear modulus.

Flow rules. As a first approximation, associated flow rules are assumed in this work:

$d \varepsilon_{\mathrm{v}}^{\mathrm{p}}=\dot{\lambda} \frac{\partial f_{\mathrm{b}}}{\partial p^{\prime}}$

$d \varepsilon_{\mathrm{q}}^{\mathrm{p}}=\dot{\lambda} \frac{\partial f_{\mathrm{b}}}{\partial q}$

where $\dot{\lambda}$ is an unknown positive scalar (often referred to as plastic multiplier) and $f_{\mathrm{b}}$ is the yield surface of the bonded material.

Effect of bonding. For the representation of the mechanical behaviour of a bonded material it is useful to consider a reference constitutive framework able to characterize the response of the unbonded material. The effect of the amount of bounds on the mechanical behaviour, can be then incorporated into this reference model. Different alternatives can be used as a reference model (e.g. González et al. 2009; González 2011; González and Gens 2011). One simple choice is to use the MCC model, as adopted in here. The existence of bonds influences the mechanical response of such reference material (i.e. unbonded) by increasing its elastic domain, its cohesion and its tensile strength. Consequently, the elastic domain of the bonded material will in general be larger in size than that corresponding to the reference model (unbonded material). This aspect is represented by Equation 7, whereas Equation 8 accounts for the increase of cohesion and tensile strength (Gens and Nova 1993):

$p_{\mathrm{c}}{ }^{\prime}=(b+1) p_{0}{ }^{\prime}$

$p_{\mathrm{t}}{ }^{\prime}=b \alpha_{\mathrm{t}} p_{0}{ }^{\prime}$

where $p_{0}{ }^{\prime}$ is the hardening parameter of the unbonded yield surface, $p_{\mathrm{c}}{ }^{\prime}$ is the hardening parameter of the bonded yield surface, $p_{\mathrm{t}}{ }^{\prime}$ relates to the increase in cohesion and tensile strength of the material with the degree of bonding, $\alpha_{\mathrm{t}}$ is a model parameter and $b$ is a non-dimensional positive variable to represent the amount of bonds in the material ( $b=0$ means no bond). Gens and Nova (1993) propose that the generation of plastic volumetric and deviatoric strains during mechanical yielding progressively reduces the influence of bonds, in such a way that the mechanical response of bonded and unbonded materials converge when all the cementation provided by the bond is lost. To give some flexibility to their approach (and allow for the possibility of a residual non-zero degree of bonding), the one-dimensional degradation law proposed by Pineda et al. (2014a) is 
adopted here. This law uses the following relationship between the damage parameter $D$ and the amount of bond $b$ :

$$
(1-D)=\frac{b}{b_{0}}
$$

where $b_{0}$ is the initial degree of bonding and $D$ is a damage parameter ranging from 0 (undisturbed state) to 1 (fully disturbed state). By assuming that the rate of damage change is proportional to the current stress state, Pineda et al. (2014a) propose the following degradation law:

$$
(1-D)=\left[\left(1-D_{0}\right)-r\right] \exp \left(-\chi d \varepsilon^{\text {damage }}\right)+r
$$

and

$$
d \varepsilon^{\text {damage }}=\left|d \varepsilon_{\mathrm{v}}^{\mathrm{p}}\right|+\left|d \varepsilon_{\mathrm{q}}^{\mathrm{p}}\right|
$$

where $D_{0}$ is the initial damage, $\chi$ is a positive parameter controlling the rate of degradation and $r$ controls the residual stiffness/tensile strength. Equation 10 governs how the effect of cementation reduces with the generation of plastic strains (i.e. increase of damage), irrespective of their sign (Equation 11). Note that both components of plastic strains (volumetric and deviatoric) are assumed to contribute to bond degradation.

Yield surfaces. The model includes two mechanical yield surfaces in the $q: p^{\prime}$ plane as (see Figure 1). A reference yield surface to represent the unbonded material $f_{\mathrm{u}}$ and a larger one for the bonded material $f_{\mathrm{b}}$. In the limit, when most bonds are being destroyed, bonded and unbonded yield surfaces progressively converge, with full convergence only if $r=0$. The reference yield surface adopted corresponds to the conventional ellipse of the MCC model, with the hardening parameter $p_{0}{ }^{\prime}$ defining its current size (Figure 1). The expression of this elliptical yield surface is given by Equation 12, where $M$ corresponds to its aspect ratio. Sheng et al. (2000) argues that the specific normalized form of Equation 12 is useful for the finite element implementation of critical state models, as this form of scaling the mathematical expression of the ellipse reduces the influences that the magnitude of stresses may have on the values of the yield surface. The larger homothetic yield surface corresponds to the bonded material. Similarly, the yield surface for the bonded material adopted is given by Equation 13, where $p_{\mathrm{c}}{ }^{\prime}$ is its hardening parameter and $p_{\mathrm{t}}{ }^{\prime}$ relates to the increase in cohesion and tensile strength given by the bond. In the limit, if $r=0$ and the effect of cementation is completely lost, the hardening parameter of the bonded material $p_{\mathrm{c}}{ }^{\prime}$ converges to $p_{0}{ }^{\prime}$, and $p_{\mathrm{t}}{ }^{\prime}$ converges to zero. If associated plasticity is assumed, plastic volumetric and deviatoric strains occur during yielding on this bonded yield surface.

$$
\begin{aligned}
& f_{\mathrm{u}}:\left(\frac{q}{p_{0}{ }^{\prime}}\right)^{2}+M^{2}\left[\left(\frac{p^{\prime}}{p_{0}{ }^{\prime}}\right)^{2}-\frac{p^{\prime}}{p_{0}{ }^{\prime}}\right]=0 \\
& f_{\mathrm{b}}:\left(\frac{q}{p_{\mathrm{c}}{ }^{\prime}}\right)^{2}+M^{2}\left[\frac{\left(p^{\prime}+p_{\mathrm{t}}{ }^{\prime}\right) p^{\prime}}{\left(p_{\mathrm{c}}\right)^{2}}-\frac{\left(p^{\prime}+p_{\mathrm{t}}{ }^{\prime}\right)}{p_{\mathrm{c}}{ }^{\prime}}\right]=0
\end{aligned}
$$



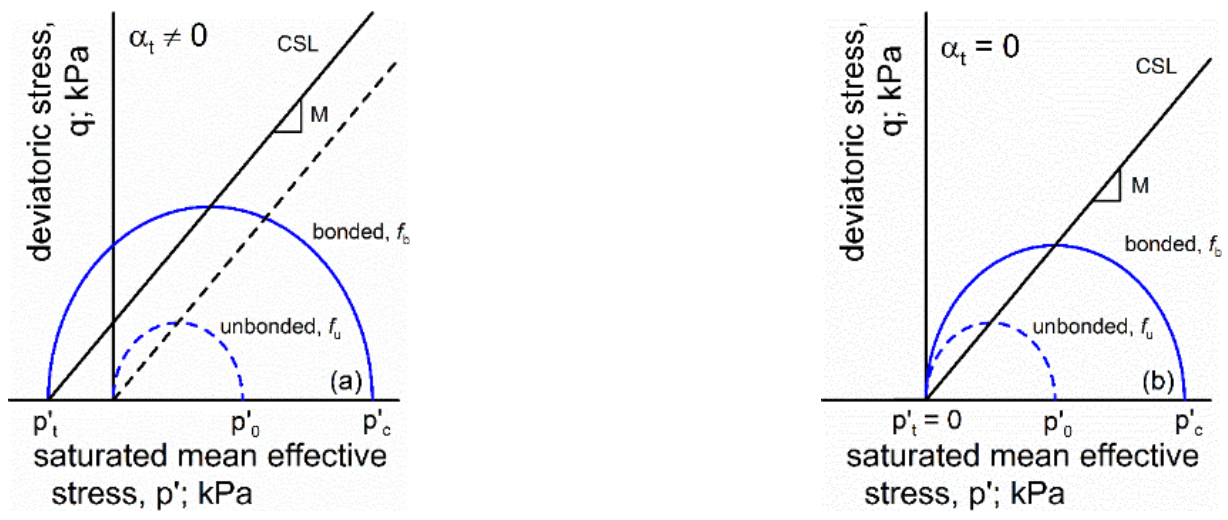

Figure 1. Yield surfaces for triaxial stress conditions: (a) effect of bonding on tensile strength; (b) no effect of bonding on tensile strength

Hardening laws. The mechanical hardening law is similar to that proposed in the MCC but includes, in addition to the effect of plastic volumetric strains, the influence of plastic deviatoric strains. Its expression is given by:

$\frac{d p_{0}{ }^{\prime}}{p_{0}{ }^{\prime}}=\frac{v}{\lambda-\kappa}\left[d \varepsilon_{\mathrm{v}}^{p}+w d \varepsilon_{\mathrm{q}}^{p}\right]$

where $\lambda$ is the gradient of a normal compression line in the $v: \ln p^{\prime}$ plane for a constant degree of cementation (such as the NCL of the unbonded material) and $w$ is a positive model parameter. It is interesting to highlight the fact that the hardening law refers to the hardening parameter corresponding to the unbonded yield surface (reference material). In this way, normal compression and critical states converge to the conventional NCL and critical state line of the MCC model when $D=1$ (and if $r=0$ ).

Consistency condition. Enforcing that the stress state remains on $f_{\mathrm{b}}$ during mechanical yielding is equivalent to impose that the total differential of $f_{\mathrm{b}}$ is zero (consistency condition):

$d f_{\mathrm{b}}=0 \Rightarrow \frac{\partial f_{\mathrm{b}}}{\partial p^{\prime}} d p^{\prime}+\frac{\partial f_{\mathrm{b}}}{\partial q} d q+\frac{\partial f_{\mathrm{b}}}{\partial p_{\mathrm{t}}{ }^{\prime}} d p_{\mathrm{t}}{ }^{\prime}+\frac{\partial f_{\mathrm{b}}}{\partial p_{\mathrm{c}}{ }^{\prime}} d p_{\mathrm{c}}{ }^{\prime}=0$

Combining Equation 15 with Equations 3-14, an expression for the unknown plastic multiplier $\dot{\lambda}$ can be obtained (see González 2011).

\section{NUMERICAL IMPLEMENTATION}

For the full implementation of a constitutive model into a finite element formulation, it is useful to program first a small subroutine responsible for the numerical integration of the stress-strain relationships of the model. These small subroutines (sometimes known as drivers) are extremely simple to use and, as shown latter in this section, are central for any preliminary check of the formulation before its final implementation into the larger finite element program. For finite 
element analysis, it is more convenient to express the incremental relationships of the model in terms of the increments of strain (instead of increments of stresses). In such case, the subroutine is referred to as strain-driven (in contrast to stress-driven formulations, where the increment of stresses are the typical variables known). Strain-driver formulations are particularly suited for finite element analysis because in these analyses, nodal displacements are usually found from the discretized global stiffness equations which are then combined with the strain-displacement relations to find the corresponding strain increments at a finite number of integration points within each element. A large number of strategies are available in the literature to perform the numerical integration of the incremental stress-strain relations of a model (Sloan 1987; Borja, 1991; Ortiz and Simo 1986, etc.). From these, the work presented here choses the family of explicit substepping algorithms with automatic error control proposed by Sloan et al. (2001).

Formulation of the problem. The notation adopted here follows that employed in Sloan et al. (2001) but making explicit the dependence of the response on the specific volume $v$, as this dependency plays a key role in the accuracy of the computed solution (Lloret-Cabot et al. 2016). The mechanical behaviour of a bonded saturated material can be characterized by the following ordinary differential equation (ODE) (with the superior dot representing a time derivative):

$$
\dot{\boldsymbol{\sigma}}^{\prime}=\mathbf{D} \dot{\boldsymbol{\varepsilon}}
$$

where $\dot{\boldsymbol{\sigma}}^{\prime}$ is a vector of effective stress rate components, $\dot{\boldsymbol{\varepsilon}}$ is a vector of strain components and $\mathbf{D}$ is the elastic matric ( $\mathbf{D}_{\mathrm{e}}$ if no plastic yielding occurs and $\mathbf{D}_{\mathrm{ep}}$ when a given strain increments causes plastic yielding). If plastic yielding occurs, two additional ODEs need to be solved accounting for the evolution of the bonded yield surface and the degree of bonding:

$$
\dot{p}_{0}{ }^{\prime}=\dot{\lambda} B_{1}
$$

$\dot{b}=b_{0}\left\{\left[\left(1-D_{0}\right)-r\right] \exp \left(-\dot{\lambda} B_{2}\right)+r\right\}-b_{0}$

where $\dot{\lambda}$ is the plastic multiplier, $B_{1}$ and $B_{2}$ are two scalar functions. Once the hardening parameter for the unbonded surface $p_{0}{ }^{\prime}$ has been updated, the corresponding hardening parameter for the bonded surface $p_{\mathrm{c}}{ }^{\prime}$ and the parameter $p_{\mathrm{t}}{ }^{\prime}$ are simply found using Equation 7 and 8, respectively. A final equation for the update of $v$ completes the system of equations:

$$
\dot{v}=-v \dot{\varepsilon}_{\mathrm{v}}
$$

Explicit substepping formulation. The system of ODEs given by Equations 16-19 defines an initial value problem IVP when the initial stress state (values of $p^{\prime}, q$ and $v$ at the start of the integration) together with the initial values of the hardening parameters $p_{0}{ }^{\prime}$ and $p_{\mathrm{c}}{ }^{\prime}$, the initial value of $p_{\mathrm{t}}{ }^{\prime}, D$ and $b$, the model parameter values and the imposed $\Delta \varepsilon$ are known. Using an explicit substepping algorithm, the solution of this IVP provides the increments of effective stresses, the updated hardening parameters and $p_{\mathrm{t}}{ }^{\prime}$, the current amount of bond and the specific volume. Details on the specific formulation of the substepping algorithm can be found elsewhere (Sloan et al. 2001; Lloret-Cabot et al. 2016). 
Numerical performance and verification aspects. Perhaps the first task once the algorithm is formulated is to verify the correctness of the numerical response during isotropic compression for $r=0$. Plotting the evolution of $v$ against $\ln p^{\prime}$ for this simple stress path allows for the verification of various numerical aspects. In particular, $v$ should vary according to a swelling compression line of gradient $\kappa$ before the stress path reaches the bonded yield surface. The stress path should reach this yield surface at $(1+b) p_{0}^{\prime}$, where a transition from elastic to elasto-plastic behaviour must be captured in the computed evolution of $v$. Beyond this yield point, the volumetric response should progressively converge to the unbonded normal compression line of gradient $\lambda$ when the cementation is not zero ( $b$ larger than one). Under such circumstances, the implemented degradation law (Equation 11) should result in a reduction of the amount of bonding available when mechanical yielding is occurring; in such a way, that (assuming $r=0$ ) the computed volumetric response for $v$ should converge to the NLC of the reconstituted material when all the bonds are entirely destroyed. This convergence ensures, in addition, that both bonded and unbonded yield surface coincide, because $p_{c}^{\prime}=p_{0}^{\prime}$ and $p_{\mathrm{t}}^{\prime}=0$ (see Equations 12 and 13). All these aspects are illustrated in Figure 2 for different degrees of bonding, including the reconstituted case i.e. $b_{0}=0$. The evolution of the specific volume during compression is illustrated in Figure $2 \mathrm{a}$ whereas Figure $2 \mathrm{~b}$ shows the degradation of the parameter $b$ when $r=0$ and no initial damage is considered.
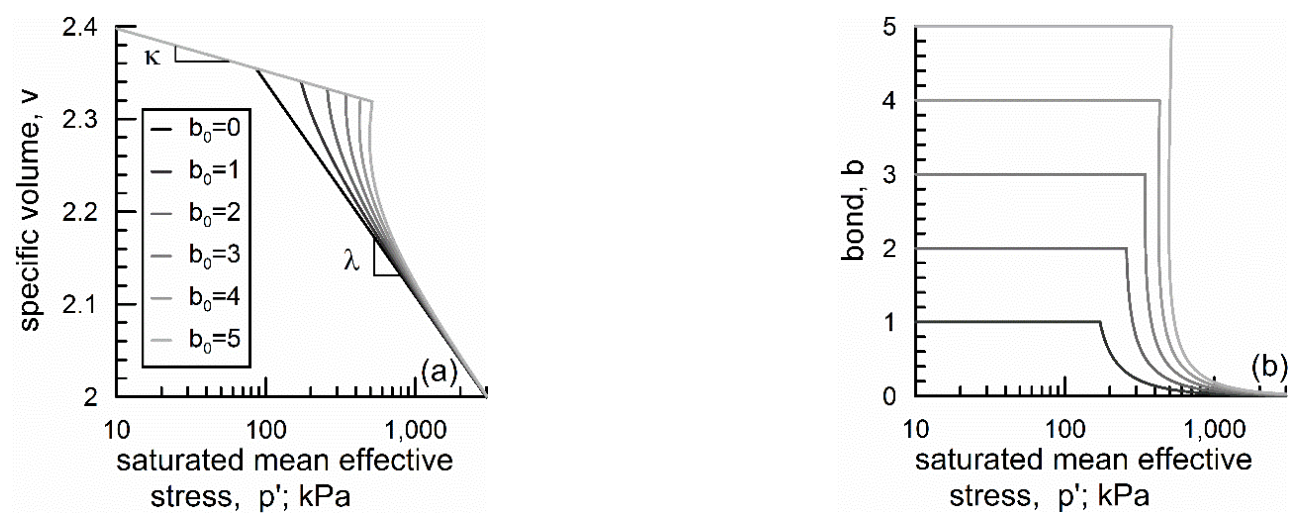

\section{Figure 2. Model responses for isotropic compression loadings at different degrees of bonding: (a) volumetric behaviour; (b) bond degradation.}

In addition to the qualitative checks discussed above, the results plotted in Figure 2 are also useful for a first assessment of the error incurred by the integration scheme. For example, gradients $\kappa$ and $\lambda$ can be obtained from a best-fitted straight line and then compared to the inputted value in the computations. Lloret-Cabot et al. (2016) propose, in this context, a more sophisticated assessment of the error behaviour incurred by a substepping integration scheme by means of plotting the socalled performance maps. This way of plotting the numerical outcomes verifies that the local error is not larger than that corresponding to the integration scheme used and is useful to assess the local and global performance of the substepping scheme. The effect of plastic deviatoric strains on 
bonding degradation cannot be evaluated in the simulations plotted in Figure 2 and, hence, other responses involving shearing stress paths should also be conducted at this stage, before full implementation of the strain-driver into the finite element program.

Comparison of the numerical solution of strain-driver and SNAC. Once the strain-driver algorithm has been implemented into the finite element program, it is convenient to verify that the solution provided by the implemented program corresponds, in fact, to the solution obtained by the strain-driver. This is illustrated in Figure 3 where the same element-test simulations showed in Figure 2 are run again as a small boundary value problem of a triaxial compression test with SNAC and the implemented model for bonded materials. As expected, Figure 3 confirms that both numerical solutions are indistinguishable for this set of isotropic tests.
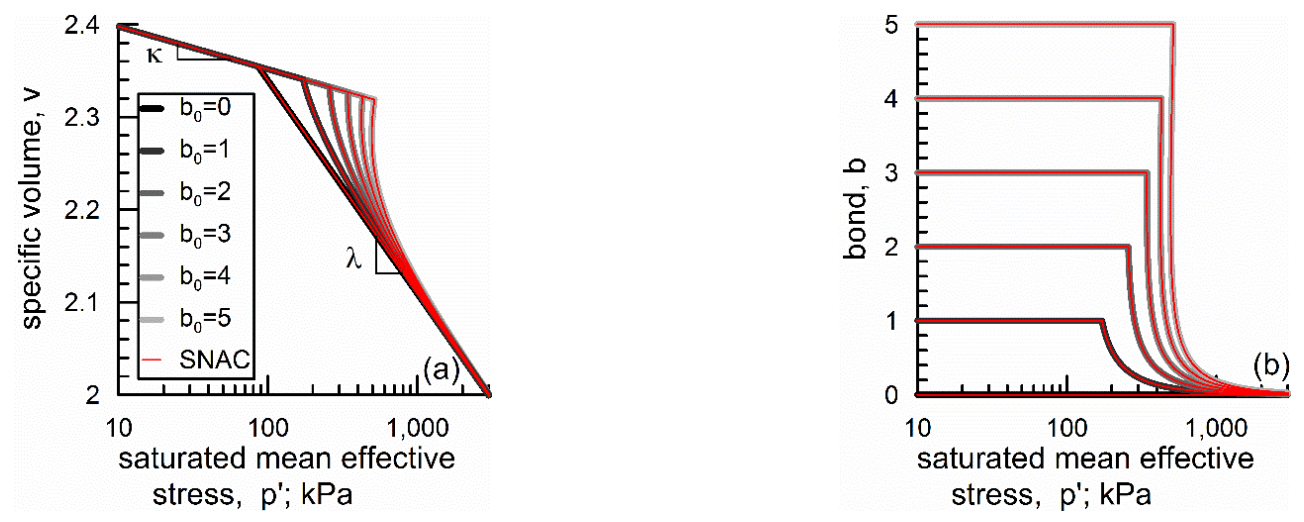

Figure 3. Numerical outcomes of strain-driver and SNAC during isotropic compression loadings at different degrees of bonding: (a) volumetric behaviour; (b) bond degradation.

\section{INFLUENCE OF THE UNSATURATED CONDITION}

It is now well accepted that the mechanical response of a bonded material under unsaturated conditions may be substantially different than that under saturated conditions (Garitte et al. 2006). Suction will often influence the mechanical properties of a bonded material. In general, when compared to a stress state at suction equal to zero, a value of suction greater than zero in a bonded material will typically show a strengthening effect and an increase of the yield stress (Leroueil \& Barbosa 2000). Additionally, a non-zero suction will also influence the mechanics of the bonds described earlier for fully saturated materials. Variations of suction during cyclic changes of relative humidity have an important impact on the mechanical properties of bonded materials as demonstrated, for example, in Pineda et al. (2014a) for an argillaceous rock (Lilla claystone, Figure 4a). Interestingly, the experimental results illustrated in Figure 4a for this material demonstrate a progressive accumulation of irreversible expansion during application of wetting-drying cycles. Figure $4 \mathrm{~b}$ shows indeed that such accumulation of swelling is what governs the degradation of stiffness moduli, tensile strength and effective cohesion. Such influence of irreversible swelling 
on all three mechanical properties is demonstrated in Pineda et al. (2014a) with the formulation of a single degradation law. The incorporation of the water retention behaviour into the constitutive relationships for saturated bonded materials presented above will be based on this set of experimental data including a degradation law consistent with these experimental observations.

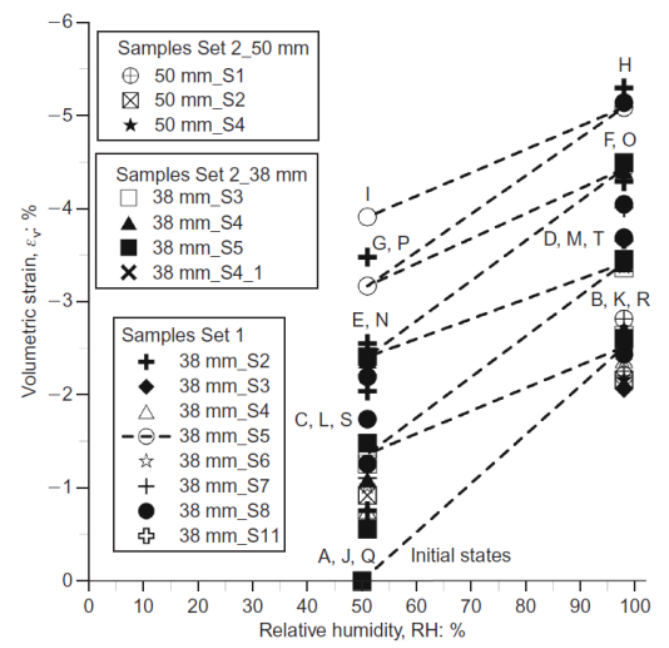

(a)

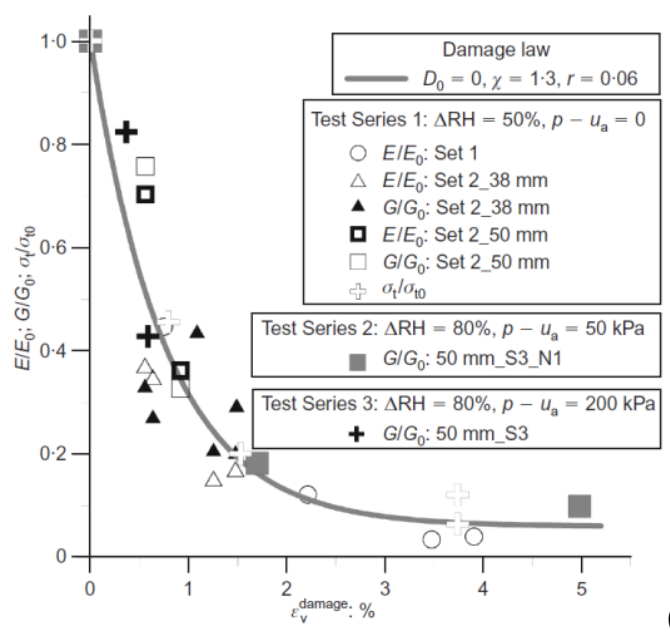

(b)

Figure 4. Degradation of Lilla claystone. (a) accumulation of volumetric expansion with the application of RH cycles. (b) degradation of stiffness moduli and tensile strength with damage volumetric strain (Pineda et al., 2014a).

\section{CONCLUSIONS}

A reference saturated model to represent the behaviour of an idealized partly or completely destructured material is the starting point to model the response of soft rocks-hard soils under saturated conditions. The combination of this reference framework with the influence that the amount of bonds has on the mechanical behaviour of bonded materials is sufficient to reproduce many relevant features of their behaviour. Some of the capabilities of such approach are demonstrated in here using the modified Cam clay and the constitutive relationships proposed in Gens and Nova (1993) for cemented materials, but incorporating the novel experimentally based degradation law proposed by Pineda et al. (2014a). The implementation of this constitutive relationships for saturated bonded materials into the finite element SNAC has been discussed in some detail highlighting the importance of the verification process. It has also been shown that the formulation of a strain-driven algorithm is particularly convenient for verification aspects, before its final implementation to a finite element program to solve boundary value problems.

\section{ACKNOWLEDGEMENTS}

This research has benefited from the "MECHANICS OF HARD SOILS AND SOFT ROCKS" (ARC-DP-2015-150103396) and “COUPLED” (MSCA-IF-2015-706712) projects. 


\section{REFERENCES}

Abbo, A.J. (1997). Finite element algorithms for elastoplasticity and consolidation. $\mathrm{PhD}$, University of Newcastle, Australia.

Borja, R.I. (1991). “Cam-Clay plasticity, Part II: Implicit integration of constitutive equation based on a nonlinear elastic stress predictor." Comp. Meth. Appl. Mech. Eng., 88, 225240.

Garitte, B., Vaunat, J. Gens, A. (2006). "A Constitutive Model that Incorporates the Effect of Suction in Cemented Geological Materials.” In $4^{\text {th }}$ International Conference on Unsaturated soils: 1944-1955.

Gens, A., Nova, R. (1993). “Conceptual bases for a constitutive model for bonded soils and weak rocks.” In Geotechnical Engineering of Hard Soils-Soft Rocks: 577-583.

González, N.A., Arroyo, M., Gens, A. (2009). "Identification of bonded clay parameters in SBPT tests: A numerical study." Soils and Found., 49(3), 329-340.

González, N.A. (2011). Development of a family of constitutive models for geotechnical applications. $\mathrm{PhD}$, Universitat Politècnica de Catalunya, Spain.

González N.A., Gens A. (2011). "Evaluation of a constitutive model for unsaturated soils: stress variables and numerical implementation.” In 5th International Conf. on Unsaturated Soils: 829-835.

Leroueil, S., Barbosa A. (2000). "Combined effect of fabric, bonding and partial saturation on yielding of soils.” In Proc. Asian Conf. On Unsaturated Soils: 527-532.

Lloret-Cabot, M., Sloan, S.W., Sheng, D., Abbo, A.J. (2016). "Error behaviour in explicit integration algorithms with automatic substepping." Int. J. Numer. Meth. Engng., 108, 1030-1053.

Lloret-Cabot, M., Wheeler, S.J., Sánchez, M. (2017a). “A unified mechanical and retention model for saturated and unsaturated soil behaviour." Acta Geotech., 12, 1-21.

Lloret-Cabot, M., Wheeler, S.J., Pineda, J.A., Romero, E., Sheng, D. (2017b). "From saturated to unsaturated conditions and vice versa." Acta Geotech., doi.org/10.1007/s11440-0170577-6.

Ortiz, M., Simo, J.C. (1986). "An analysis of a new class of integration algorithms for elastoplastic constitutive relations.” Int. J. Numer. Meth. Engng., 23, 353-366.

Pineda, J.A., Alonso, E.E., Romero, E. (2014a). "Environmental degradation of claystones", Géotechnique, 64(1), 64-82.

Pineda, J.A., Romero, E., De Gracia, M., Sheng, D. (2014b). "Shear strength degradation in claystones due to environmental effects." Géotechnique, 64, 493-501.

Roscoe, K.H., Burland, J.B. (1968). "On the generalised stress-strain behavior of wet clay." Engineering Plasticity (eds Heyman J \& Leckie FA), Cambridge University Press, Cambridge: 535-609. 
Sloan, S.W. (1987). "Substepping schemes for the numerical integration of elastoplastic stressstrain relations." Int. J. Numer. Meth. Engng., 24, 893-911.

Sloan, S.W., Abbo, A.J. (1999a). "Biot consolidation analysis with automatic time stepping and error control Part I: Theory and Implementation." Int. J. Numer. Anal. Meth. Geomech., 23, 467-492.

Sloan, S.W., Abbo, A.J., Sheng, D. (2001). "Refined explicit integration of elastoplastic models with automatic error control.” Eng. Comp.; 18(1-2), 121-154. Erratum: Eng. Comp. 2002, 19(5-6), 594-594.

Sheng, D., Sloan, S.W., Yu, H.S. (2000). "Aspects of finite element implementation of critical state models." Comp. Mech., 26: 185-196.

Sheng, D., Sloan, S.W., Gens, A., Smith, D.W. (2003). "Finite element formulation and algorithms for unsaturated soils. Part I: Theory." Int. J. Numer. Anal. Meth. Geomech.; 27, 745-765. 